\title{
Edge Colorings of Complete Graphs Without Tricolored Triangles
}

\author{
András Gyárfás ${ }^{1}$ and Gábor Simonyi ${ }^{2}$ \\ ${ }^{1}$ COMPUTER AND AUTOMATION RESEARCH INSTITUTE \\ OF THE HUNGARIAN ACADEMY OF SCIENCES \\ BUDAPEST, P.O. BOX 63, 1518, HUNGARY \\ E-mail:gyarfas@sztaki.hu \\ ${ }^{2}$ ALFRED RÉNYI INSTITUTE OF MATHEMATICS \\ HUNGARIAN ACADEMY OF SCIENCES \\ BUDAPEST, P.O. BOX 127, 1364, HUNGARY \\ E-mail: simony@renyi.hu
}

Received June 3, 2002; Revised November 4, 2003

DOI 10.1002/jgt.20001

\begin{abstract}
We show some consequences of results of Gallai concerning edge colorings of complete graphs that contain no tricolored triangles. We prove two conjectures of Bialostocki and Voxman about the existence of special monochromatic spanning trees in such colorings. We also determine the size of largest monochromatic stars guaranteed to occur.

(c) 2004 Wiley Periodicals, Inc. J Graph Theory 46: 211-216, 2004
\end{abstract}

Keywords: Gallai coloring; monochromatic spanning tree; Ramsey theory

Contract grant sponsor: OTKA (to A.G.); Contract grant number: T029074; Contract grant sponsor: OTKA (to G.S.); Contract grant numbers: T032323, T037486.

(C) 2004 Wiley Periodicals, Inc. 


\section{INTRODUCTION}

We consider edge colorings of complete graphs in which no triangle is colored with three distinct colors. These colorings generalize 2-colorings and we shall call them Gallai colorings. A similar terminology, Gallai partition, is used in Ref. [10]. The reason is the close connection of these colorings to the basic work [8] of Gallai on comparability graphs. Gallai colorings also appear in Ref. [5], a paper of Cameron, Edmonds and Lovász where the (weak) perfect graph theorem [11] is extended (see Theorem $\mathrm{C}$ below). They turned out to be relevant also in investigations concerning the additivity properties of the information theoretic functional called graph entropy (see Refs. $[9,10]$ ).

In this paper we look at some Ramsey-type problems for Gallai colorings. The first problems of this type were studied by Erdôs, Simonovits and Sós in Ref. [7] where it was shown that Gallai colorings of $K_{n}$ can use at most $n-1$ colors (see Proposition B). Equality holds for the coloring where, for $i=1,2, \ldots, n-1$ color $i$ forms a star of $i$ edges. This shows that a Gallai coloring may be such that all of its monochromatic subgraphs are stars. Gallai colorings, like (their special case of) 2-colorings, always have monochromatic spanning trees. This is observed by Bialostocki, Dierker and Voxman in [1]. In Ref. [2] (also in Ref. [1]) Bialostocki and Voxman raise three problems about the existence of specific monochromatic spanning trees in Gallai colorings. We answer these problems (two of them positively) as follows. In any Gallai coloring, there is a monochromatic spanning broom, where a broom is a path with a star at its end (Theorem 2.1). Burr (in Ref. [3]) proved this for 2-colorings (conjectured also by Bialostocki). Gallai colorings also contain monochromatic spanning trees of height two (Theorem 2.2). We also prove that the largest monochromatic star which must appear in any Gallai coloring of $K_{n}$ has at least $2 n / 5$ edges (Theorem 3.1). An easy construction shows that this bound is sharp, implying a negative answer to the third question of Bialostocki and Voxman.

It is obvious that Gallai colorings are closed under substitution: replacing a vertex in a Gallai coloring by a complete graph with a Gallai coloring gives again a Gallai coloring. The following important result shows that all Gallai colorings can be obtained by substituting into 2-colored complete graphs. Theorem A (and Lemma A) is implicit in Ref. [8] and also among the results of Cameron and Edmonds on Lambda composition (see Ref. [4]). Due to its importance (and to keep the paper self-contained) we state and prove it.

Theorem A. Any Gallai coloring can be obtained by substituting complete graphs with Gallai colorings into vertices of 2-colored complete graphs.

Theorem A can be applied to extend results from 2-colorings to Gallai colorings. We shall refer to the 2-colored complete graph as the base graph and the graphs substituted into the vertices of the base graph will be called the blocks.

Theorem A will be derived from the following property of Gallai colorings which is essentially the content of Lemma (3.2.3) in Ref. [8]. 
Lemma A. Every Gallai coloring with at least three colors has a color which spans a disconnected graph.

Now Theorem A is obvious from Lemma A: If a Gallai coloring is just a 2-coloring, we are done. Otherwise we have a color with at least two components. It is clear that edges between any two components are colored with the same color. Collapsing the components into vertices, we have a smaller graph with a Gallai coloring which, by induction, can be generated as required.

Proof. Let $G$ be a minimal counterexample, clearly all colors appear on some edge incident to any particular vertex of $G$. Let $x \in V(G)$ and $H=G \backslash x$. Then $H$ cannot be 2-colored because then any other color would span a disconnected graph. By minimality, $H$ is disconnected in some color, say in color 1 with components $C_{1}, \ldots, C_{k}$. As noted before, all edges between any fixed pair of components have the same color (different from 1).

We claim that $G$ is disconnected in color 1 . Indeed, assume that there are edges of color 1 from $x$ to $y_{i} \in C_{i}$ for every $i$. Let $x u$ and $x v$ be edges of color 2 and 3 .

Case 1. If $u, v$ are in the same component, say $u, v \in C_{1}$ then $u y_{2}$ must be of color 2 and $v y_{2}$ must be of color 3 (using that the triangles $x u y_{2}$ and $x v y_{2}$ are not tricolored). This contradicts the homogeneous coloring of the edges between $C_{1}$ and $C_{2}$.

Case 2. If $u, v$ are in different component, say $u \in C_{1}, v \in C_{2}$ then $u y_{2}$ must be of color 2 and $v y_{1}$ must be of color 3 (using that the triangles $x u y_{2}$ and $x v y_{1}$ are not tricolored). We get the same contradiction as in Case 1.

Therefore, the claim is proved, $G$ is disconnected in color 1 . Thus $G$ cannot be a counterexample.

Theorem A can be conveniently used to derive properties of Gallai colorings. The following result is from Ref. [7].

Proposition B. At most $n-1$ colors can be used in any Gallai coloring of $K_{n}$.

Proof. Apply induction for the blocks of the base graph.

Theorem A can also be used to give the following generalization of the (weak) perfect graph theorem. The theorem is from Ref. [5], its relation to Gallai's work is further emphasized in Ref. [4]. (See Ref. [10] for a generalization of Theorem $\mathrm{C}$ where Lemma A also plays an important role.)

Theorem C. If all but one color classes of a Gallai coloring span perfect graphs then all color classes span perfect graphs.

\section{MONOCHROMATIC SPANNING TREES IN GALLAI COLORINGS}

An old remark of Paul Erdős says that 2-colored complete graphs have monochromatic spanning trees. One can also say something about the type of the 
spanning tree. Bialostocki, Dierker, and Voxman proved in Ref. [1] that there is a monochromatic spanning tree of height at most two. Burr [3] proved, answering the conjecture of Bialostocki, that there is a spanning 'broom,' which means the union of a path and a star with the central vertex of the latter identified with an endvertex of the former. Bialostocki and Voxman conjectured (Ref. [2], Problems $3.3 \mathrm{a}$ and c) that both results can be generalized to Gallai colorings. Theorems 2.1 and 2.2 verify these conjectures using Theorem A. The essential steps in the proof of Theorem 2.1 follow Burr's nice (unpublished) proof [3].

Theorem 2.1. In every Gallai coloring of a complete graph $K$ there is a monochromatic spanning broom.

Proof. Using Theorem A, we assume that the base graph of $K$ is colored with colors red and blue. Without loss of generality, assume that the red edges determine a $k$-connected graph and the blue edges determine an at most $k$ connected graph on the vertex set of $K$ ( $k$ is a positive integer). This implies that there is a subset $A$ with $|A| \leq k$ whose removal disconnects the blue graph. We may also assume that $|A|$ is as small as possible, i.e., $A$ is a minimal separator of the blue graph. If $A$ is empty, i.e., the blue graph is disconnected, then the vertices of $K$ are spanned by a red complete bipartite graph which obviously contains a red spanning broom. Therefore, $A$ is nonempty. By definition, $V(K) \backslash A$ has a nontrivial partition into $X, Y$ such that there are no blue edges between $X$ and $Y$.

Claim 2.1. $X \cup Y$ has a red spanning complete bipartite graph $H$.

If there are no vertices $x \in X$ and $y \in Y$ such that $x, y$ belong to the same block of the base graph then all edges between $X$ and $Y$ are red and the claim is proved. Otherwise there is a block $B$ of the base graph such that $U=B \cap X, V=B \cap Y$ are nonempty. It follows that all edges between $U \cup V$ and $(X \cup Y) \backslash(U \cup V)$ are red-unless $U=X$ and $V=Y$. However, in this case every vertex of $A \backslash B$ sends a blue edge to $B$, thus all edges between $B$ and $A \backslash B$ are blue. This implies that the base graph is disconnected in red, a contradiction. Thus the claim is proved.

Now the proof is finished by applying a well-known result of Dirac [6] which says that any $k$ vertices of a $k$-connected graph can be covered by a cycle of at least $k+1$ vertices. We use this theorem for the $k$-connected red graph and the $k$ vertices in $A$. Let the cycle guaranteed to exist by Dirac's theorem be $C$. (In the degenerate case when $k=1, C$ is defined as a red edge containing the vertex of A.) Thus the vertex set of $K$ is covered by $C \cup H$. Using that $C$ and $H$ have nonempty intersection one can easily find a red spanning broom.

Theorem 2.2. In every Gallai coloring, there is a monochromatic spanning tree with height at most two.

Proof. By Theorem A, the Gallai coloring can be obtained by substitutions into a 2-colored base graph $H$. It is easy to see (cf. Theorem 2.1 in Ref. [1]) that $H$ has a monochromatic spanning tree $T$ with height at most two (the root can be any vertex with maximum monochromatic degree). One can easily extend $T$ : 
substituting a set $X$ into a nonroot vertex $x$ of $T$ results in adding each element of $X$ as a leaf with the same father; substituting into the root of $T$ results in adding each element but one (that remains the root) of $X$ as a leaf with its father at an arbitrary vertex of level one in $T$.

\section{MONOCHROMATIC STARS IN GALLAI COLORINGS}

It is a natural question to ask for the maximum monochromatic degree in a Gallai coloring of $K_{n}$. Consider the red-blue coloring of $K_{5}$ where both color classes form pentagons. Substituting green complete graphs into this base graph, one can get a Gallai colored $K_{n}$ with no monochromatic degree exceeding $2 n / 5$. This construction is best possible as shown by the next theorem (and provides a negative answer to Problem 3.3b in Ref. [2]).

Theorem 3.1. Any Gallai coloring of $K_{n}$ has a color with largest degree at least $2 n / 5$.

Proof. By Theorem A, the Gallai coloring can be defined by substituting into a base graph colored with colors 1,2 . It is easy to check that if the base graph has at most four vertices then color 1 or 2 has degree at least $n / 2$. If the base graph has at least five vertices then there is a block $B$ with at most $n / 5$ vertices. Therefore, any vertex in $B$ is adjacent to at least $4 n / 5$ vertices outside $B$ in colors 1 or 2 and the theorem follows.

One can use Theorem 3.1 in the proof of Theorem 2.2 to show that the root of the monochromatic spanning tree found there can be of degree at least $2 n / 5$.

\section{REFERENCES}

[1] A. Bialostocki, P. Dierker, and W. Voxman, Either a graph or its complement is connected: A continuing saga (manuscript in preparation).

[2] A. Bialostocki and W. Voxman, On monochromatic-rainbow generalizations of two Ramsey-type theorems. Ars Combinatoria 68 (2003), 131-142.

[3] S. A. Burr, Either a graph or its complement contains a spanning broom (manuscript in preparation).

[4] K. Cameron and J. Edmonds, Lambda composition, J Graph Theory 26 (1997), 9-16.

[5] K. Cameron, J. Edmonds, and L. Lovász, A note on perfect graphs, Period Math Hungar, 17 (1986), 173-175.

[6] G. A. Dirac, In abstrakten Graphen vorhandene vollständige 4-graphen und ihre Unterteilungen, Math Nachr 22 (1960), 61-85.

[7] P. Erdós, M. Simonovits, and V. T. Sós, Anti-Ramsey theorems, Coll Math Soc J Bolyai 10 (1973), 633-643. 
[8] T. Gallai, Transitiv orientierbare Graphen, Acta Math Acad Sci Hungar 18 (1967), 25-66. English translation by F. Maffray and M. Preissmann, In: J. L. Ramírez-Alfonsín and B. A. Reed, (editors), Perfect Graphs, John Wiley and Sons, 2001, pp. 25-66.

[9] J. Körner, G. Simonyi, Graph pairs and their entropies: Modularity problems, Combinatorica 20 (2000), 227-240.

[10] J. Körner, G. Simonyi, and Zs. Tuza, Perfect couples of graphs, Combinatorica 12 (1992), 179-192.

[11] L. Lovász, Normal hypergraphs and the perfect graph conjecture, Discrete Math 2 (1972), 253-267. 